\title{
QUEEN'S
UNIVERSITY
BELFAST
}

\section{Development of a nanoarray capable of the rapid and simultaneous detection of zearalenone, T2-toxin and fumonisin}

McNamee, S. E., Bravin, F., Rosar, G., Elliott, C. T., \& Campbell, K. (2017). Development of a nanoarray capable of the rapid and simultaneous detection of zearalenone, T2-toxin and fumonisin. Talanta, 164, 368-376. https://doi.org/10.1016/j.talanta.2016.11.032

\section{Published in:}

Talanta

\section{Document Version:}

Peer reviewed version

Queen's University Belfast - Research Portal:

Link to publication record in Queen's University Belfast Research Portal

\section{Publisher rights}

(c) 2017 Elsevier Ltd. This manuscript version is made available under the CC-BY-NC-ND 4.0 license http://creativecommons.org/licenses/bync-nd/4.0/ which permits distribution and reproduction for non-commercial purposes, provided the author and source are cited.

\section{General rights}

Copyright for the publications made accessible via the Queen's University Belfast Research Portal is retained by the author(s) and / or other copyright owners and it is a condition of accessing these publications that users recognise and abide by the legal requirements associated with these rights.

Take down policy

The Research Portal is Queen's institutional repository that provides access to Queen's research output. Every effort has been made to ensure that content in the Research Portal does not infringe any person's rights, or applicable UK laws. If you discover content in the Research Portal that you believe breaches copyright or violates any law, please contact openaccess@qub.ac.uk. 
Development of a nanoarray capable of the rapid and simultaneous detection of zearalenone, T2-toxin and fumonisin

Sara E. McNamee ${ }^{1}$, Francesca Bravin ${ }^{2}$, Giulia Rosar $^{2}$, Christopher T. Elliott ${ }^{1}$, Katrina Campbell $^{1^{*}}$

${ }^{1}$ Institute for Global Food Security, School of Biological Sciences, Queen's University, Stranmillis Road, Belfast, BT9 5AG, UK

${ }^{2}$ Tecna s.r.l., c/o Area Science Park, Località Padriciano, 99 - 34149 Trieste, Italy.

\section{* Corresponding author}

Dr Katrina Campbell

Institute for Global Food Security, School of Biological Sciences, Queen's University, David Keir Building, Stranmillis Road, Belfast, UK BT9 5AG

Telephone: +44 (0) 2890976535

Fax: +44 (0) 2890976513

Email: katrina.campbell@qub.ac.uk 


\section{Abstract}

Fusarium mycotoxins such as trichothecenes, zearalenone and fumonisins occur on a worldwide basis in cereal grains, animal feeds and forages. Practical solutions for multiple mycotoxin determination in samples are required by industry and regulators for cost effective screening purposes. The feasibility of developing a novel multiplex nanoarray for the simultaneous and semi-quantitative detection of three regulated mycotoxins: zearalenone (ZEA), T2-toxin (T2) and fumonisin B1 (FUM) was examined. Additionally, the assay was also able to detect HT2 toxin and fumonisin B2 and B3 due to the cross reactivity profiles of the antibodies used. Individual mycotoxin conjugates specific to the three mycotoxins were nano-spotted onto wells of a microtitre plate. Optimisation of assay parameters and antibodies was undertaken with both individual and multiplex calibration curves generated. A competitive assay format was employed enabling a calibration curve for concentration analysis and duplicate results for up to 40 samples in $70 \mathrm{~min}$ for the three target mycotoxins. The characteristics and performance of the nanoarray were evaluated including sensitivity and specificity for each target. Additionally, intra and inter spotting precision, cross reactivity, matrix effects and sample analysis in maize and wheat $(n=8)$ was performed. Sensitivity, determined as the concentration causing $50 \%$ inhibition, was 70.1, 2.8 and $90.9 \mathrm{ppb}$ in PBS, 172.4, 3.2 and 129.3 ppb in methanol, 197.4, 0.7 and 216.7 ppb in wheat and 43.6, 0.5 and 25.9 ppb in maize for ZEA, T2 and FUM respectively. Intra spotting precision was 6, 11 and $10 \%$ for PBS and 5, 11 and $12 \%$ for methanol for ZEA, T2 and FUM respectively. Inter spotting precision was 4, 14 and $6 \%$ for PBS and 3, 9 and $16 \%$ for methanol for ZEA, T2 and FUM respectively. The feasibility of the nanoarray as an easy to use sensitive screening tool in the 96 well format has been demonstrated for the multiplex detection of three regulated mycotoxins. Improvements in automated image and data analysis software for novice end users are required to improve the overall rapidity of analysis. 
Keywords:

Mycotoxin; nanoarray; multiplex; zearalenone; T2-toxin; fumonisin

\section{Introduction}

Mycotoxins are naturally occurring secondary chemical metabolites produced by different fungi genera such as Aspergillus, Fusarium, Penicillium, Alternaria and Claviceps [1]. Among these genera, Fusarium fungi are the most widespread in cereal crop production. Fusarium fungi produce a diversity of mycotoxin types and can have widespread geographical distribution influenced primarily by environmental and climatic conditions and crop production, storage, processing and transportation methods. Toxins produced by Fusarium moulds, include fumonisins, trichothecenes (deoxynivalenol, T2 and HT2 toxin) and zearalenone. Mycotoxins are chemically and thermally stable whereby mycotoxin contamination of raw materials may also affect processed foods [2]. Furthermore they can enter the food chain through animals fed contaminated feed [3]. Mycotoxin contamination is inhomogeneous in nature and the toxins can be present at very low levels. Due to their highly resistant nature they can remain in the food chain [4] thereby posing a threat as toxic contaminants of food products.

The complex toxic effects of mycotoxins pose significant health risks to humans and animals and this necessitates for effective control and surveillance procedures [5-9]. The initial stages in the protection against mycotoxin contamination is through the adoption of good agricultural, storage and processing practices to ensure mycotoxin levels remain negligible or as low as reasonably achievable [10]. In order to protect consumer safety, legislative limits for mycotoxins in certain foodstuffs are set out by the European Commission [11-14].

Contamination of food by these natural toxins is of an increasing safety and economic concern due to changes in prevalence with variations in environmental conditions. Therefore, 
innovation in the development of detection methods for implementation is vitally important. The detection of mycotoxins is carried out by confirmatory methods for quantitative analysis and by rapid diagnostics for screening. Several validated methods are available for the measurement of mycotoxins and the literature on this subject has included many comprehensive and critical reviews [8, 15-19]. For the quantitative analysis of mycotoxins high performance liquid chromatography (HPLC), gas chromatography (GC) and thin layer chromatography (TLC) as well as multiple toxin mass spectrometry (MS) methods are used [20-24]. These methods, however, require the use of complex and expensive equipment as well as skilled operators and extensive sample pre-treatment. Furthermore, these methods sometimes may not reach the very low limit of detection required. Immunoassays are often employed for the screening of mycotoxin contamination as they provide rapid, sensitive detection and easy to use methods. Immunological methods such as enzyme linked immunosorbent assay (ELISA) [25-29] and lateral flow devices (LFD) [30-33] are the most common approaches. These detection methods are simpler in design, inexpensive, fast, robust, user friendly and cost effective allowing high sample throughput with high sensitivity and accuracy. LFDs provide a 'yes/no' answer, however, in recent years modified devices enabling quantitative evaluation and multiplexing have been developed. In the Conffidence project funded by the European Union, a LFD enabling the simultaneous detection of Fusarium toxins (deoxynivalenol, zearalenone, T2/HT2 toxins, fumonisins) in cereals was developed [34] and now commercialised by Unisensor (Belgium). Commercial detection tests based on both ELISA and LFDs are also available for many of the mycotoxins from Tecna (Italy), RBiopharm (Germany), Europroxima (The Netherlands), Neogen (USA), Charm Sciences (USA), Diachemix (USA), Envirologix (USA), Romer Labs (USA) and Vicam (USA) but are all based on single toxin analysis for these mycotoxins. Randox (Crumlin, UK) offer a 
commercial test for multiple mycotoxin detection based on a customised biochip with chemiluminescent detection using relatively expensive closed technology.

In addition to safety issues, natural toxin contamination of food is of great economic concern; much effort is therefore devoted to the development of novel, rapid, inexpensive, simple and sensitive multiple mycotoxin screening methods. The simultaneous identification of several mycotoxins in one single test, reducing time and costs per analysis is a most attractive option. Nanoarrays are important tools for high throughput analysis enabling miniaturization, higher sensitivity and simplified sample preparation and offer a number of applications in the areas of medical diagnosis, genetic testing, environmental monitoring and food safety. In recent years the nanoarray format has provided a powerful tool in which several targets are separately detected in spatially defined zones simultaneously. Oswald et al. (2013) described a multiple mycotoxin immunoassay using the Munich chip reader 3 platform and reusable biochips. A number of mycotoxins including aflatoxin, ochratoxin A, FUM and DON were spotted onto glass slides and detected in cereals using chemiluminescence [35]. Additionally, Beizaei et al. (2015) reported a rapid and highly sensitive microarray method for aflatoxin $\mathrm{B}_{1}$ detection in cereals using 16-pad nitrocellulose coated FAST slides [36]. The application of nanotechnology faces many challenges in order to produce successful commercial products that can compete with the traditional methods of mycotoxin analysis. In this study, it is intended to introduce the concept and advantages of nanotechnology to the food industry and describe the proof of concept and feasibility of a nanoarray for the simultaneous detection of three harmful mycotoxins. There are very few studies that effectively employ this promising technology for the detection of mycotoxins. Innovative nano science and technology with state of the art sensing equipment have allowed novel detection methods to become a reality. The aim of this research was therefore to develop a multiplex nanoarray for the simultaneous 
detection of three regulated Fusarium mycotoxins offering high throughput in the 96 well plate format compared to LFD platforms.

3. Materials and methods

3.1. Instrumentation

A sciFLEXARRAYER S5 (Scienion, Germany) was used for spotting microtitre plates and a sciReader CL colorimetric nanoarray reader (Scienion, Germany) was used for scanning and analysing spot intensities.

\subsection{Reagents}

Antibodies for ZEA, T2 and FUM and mycotoxin-BSA conjugates for ZEA (3.39 mg/ml), T2 $(1.5 \mathrm{mg} / \mathrm{ml})$ and FUM $(1.32 \mathrm{mg} / \mathrm{ml})$ were provided by Tecna (Tecna s.r.l, Trieste, Italy). Nunc 96 well microtitre plates were purchased from VWR (Leicestershire, UK). Alkaline phosphatase substrate was purchased from Millipore (Hertfordshire, UK). Bovine serum albumin (BSA), anti-rabbit IgG-alkaline phosphatase antibody produced in goat, 5-bromo-4chloro-3'-indolyphosphate (BCIP), nitro-blue tetrazolium (NBT), methanol (HPLC grade), zearalenone, deoxynivalenol and HT2-toxin were all purchased from Sigma-Aldrich (Dorset, UK). Fumonisin B1 was purchased from Trilogy (Darmstadt, Germany). T2-toxin, fumonisin B2 and fumonisin B3 were purchased from Romer Labs (Cheshire, UK).

\subsection{Printing nanoarrays}

BSA-conjugated mycotoxins were diluted in filtered printing buffer (100 mM sodium phosphate, $50 \mathrm{mM}$ sodium chloride, $100 \mu \mathrm{g} / \mathrm{ml} \mathrm{BSA,} 0.005 \%$ Tween-20, pH 8.0) at $50 \mu \mathrm{g} / \mathrm{ml}$. A spotting volume of $1000 \mathrm{pl}$ for each reagent was spotted onto a 96 well microtitre plate using a sciFLEXARRAYER S5. For part one of the study (single spotting analysis) a nine spot matrix 
format (3x3 array) was arrayed with a $1000 \mu \mathrm{m}$ spot to spot pitch composing of nine replicates of each target in separate wells. For part two of the study (multi spotting analysis) a 12 spot matrix format (4x3 array) was arrayed with a $750 \mu \mathrm{m}$ spot to spot pitch composing of four replicates of each target (x 3 targets) in the same well. All spotting was carried out at room temperature and $65 \%$ humidity. Microtitre plates were left at $65 \%$ humidity for $1 \mathrm{hr}$ on the nanospotter before being stored at $25^{\circ} \mathrm{C}$ and $30 \%$ humidity overnight in a humidity chamber (Deny, China).

\subsection{Assay protocol}

The microtitre plate was blocked with $0.2 \% \mathrm{BSA}(100 \mu \mathrm{l})$ for $60 \mathrm{~min}$ at room temperature followed by 3 washes with ELISA wash solution (0.15 M NaCl, $0.0125 \%$ Tween $)$ and dried on lint free paper. Antibody (50 $\mu \mathrm{l})$ diluted in PBS (0.1 M, pH 7.2) and sample/standard (50 $\mu 1)$ diluted in PBS (0.1 M, pH 7.2) were applied to each well and incubated for $40 \mathrm{~min}$ at room temperature. The microtitre plate was washed 3 times with ELISA wash solution and dried with lint free paper. Alkaline phosphatase anti-rabbit IgG $(100 \mu \mathrm{l})$ diluted 1/500 in PBS $(0.1$ $\mathrm{M}, \mathrm{pH}$ 7.2) was added to each well and incubated for $20 \mathrm{~min}$ at room temperature. The microtitre plate was washed 3 times with ELISA wash solution and dried with lint free paper. BCIP/NBT substrate $(100 \mu \mathrm{l})$ was added to each well and incubated for $10 \mathrm{~min}$ at room temperature. Finally, the microtitre plate was washed 3 times with ELISA wash solution and dried with lint free paper.

\subsection{Image and data processing}

Microtitre plates were scanned using the sciReader colorimetric nanoarray reader at an exposure of $100 \mathrm{~ms}$. An image of each well of the microtitre plate was taken and saved as a TIFF file. One microtitre plates takes approximately 1 min to scan. The images are opened and 
processed using sciANA software from Scienion. The spotting matrix of each well is defined by the number of blocks $(1 \times 1)$ and number of spots $(4 \times 3)$ so that the software knows the spotting configuration. Next the image is evaluated and the software attempts to find the spotting configuration that has been specified. If the software successfully detects the spots it will draw grids around each spot automatically. If the intensity between the spots and the background is similar the software will not be able to find the spots and instead the grids must be manually aligned by the user. Once grids are aligned the data can be exported into excel. The excel sheet will contain information on each spot of the well including for example $\mathrm{X}$ and Y coordinates, diameter, median intensity of spot and intensity of background. The median intensity (with background removed) measured in pixels is used for further data analysis.

\subsection{Optimisation}

A chequerboard design was employed to optimise the assay parameters. Microtitre plates were spotted in a single system (ZEA, T2 or FUM) to determine optimum parameters for each mycotoxin. Single antibodies to each mycotoxin were assessed. For ZEA microtitre plates were spotted at varying spotting volumes $(330,670,1000,2000 \mathrm{pl})$ at varying protein concentrations $(0.5,5$ and $50 \mu \mathrm{g} / \mathrm{ml})$ of the ZEA-BSA conjugate. For T2 and FUM a spotting volume of 1000 $\mathrm{pl}$ at varying protein concentrations of $10,50,100$ and $200 \mu \mathrm{g} / \mathrm{ml}$ of the mycotoxin-BSA conjugates were assessed. Two antibodies were compared for T2 (ABT-1 and ABT-2) and FUM (ABF-1 and ABF-2) while only one antibody was available for ZEA (ABZ). These were assessed at different dilutions ranging from 1/500 - 1/15,000. A negative and positive standard were assessed for each parameter to determine optimum spotting conditions for each mycotoxin. 


\subsection{Individual calibration curves}

Microtitre plates were spotted in a single system (ZEA, T2 or FUM) to assess individual calibration curves for each mycotoxin. Spotting parameters and the assay parameters were determined during the optimisation stage. The microtitre plates were spotted using mycotoxinBSA conjugates at a spotting volume of $1000 \mathrm{pl}$ and a protein concentration of $50 \mu \mathrm{g} / \mathrm{ml}$ for all three mycotoxin-BSA conjugates. Single antibodies for ZEA ABZ (1/10,000), T2-ABT-1 (1/4000) T2-ABT-2 (1/4000), FUM-ABF-1 (1/4000) and FUM-ABF-2 (1/4000) were examined during the assay. Eight point calibration curves for ZEA, T2 or FUM were prepared in PBS (0.1 M, pH 7.2) and $23.3 \%$ methanol (in water v/v) at concentrations across the full dynamic range for a full evaluation for individual mycotoxin analysis. The methanol curve (23.3\%) was included throughout for comparison as the final extract in matrix (maize and wheat) was optimised providing $23.3 \%$ as the final percentage methanol in samples applied to the assay. Individual calibration curves were assessed and examined ( $\mathrm{n}=2$ analysis, 9 spots per analysis) and sensitivity was assessed using the $50 \%$ inhibition concentration. The $50 \%$ inhibition concentrations were determined from a 4 parameter fit curve using BIAevaluation version 4.1 software (Biacore, GE Healthcare).

\subsection{Multiplex calibration curves}

Microtitre plates were spotted in a three-plex system (ZEA, T2 and FUM) to assess multiplex calibration curves for each mycotoxin. Spotting and assay parameters were determined during the optimisation stage. Microtitre plates were spotted using mycotoxin-BSA conjugates at a spotting volume of $1000 \mathrm{pl}$ and a protein concentration of $50 \mu \mathrm{g} / \mathrm{ml}$ for all three mycotoxinBSA conjugates. Antibodies for ZEA (ABZ 1/10,000), T2 (ABT-1 1/4000) and FUM (ABF-2 1/4000) were examined during the assay. Eight point calibration curves for ZEA, T2 and FUM were prepared in PBS and methanol at concentrations across the full dynamic range for a full 
evaluation for individual mycotoxin analysis. Where necessary additional standards were added to improve the curve shape. Multiplex calibration curves were assessed and examined ( $n=6$ analysis, 4 spots per analysis, 3 spotting days) and sensitivity was assessed using the 50 $\%$ inhibition concentration. The $50 \%$ inhibition concentrations were determined from a 4 parameter fit curve using BIAevaluation version 4.1 software (Biacore, GE Healthcare).

\subsection{Cross reactivity}

Microtitre plates were spotted as described in section 3.7. Antibodies for ZEA (ABZ 1/10,000), T2 (ABT-1 1/4000) and FUM (ABF-2 1/4000) were examined during the assay for ZEA, T2, HT2, FUM B1, FUM B2, FUM B3, DON as individual calibration curves. Eight point calibration curves were prepared in methanol at concentrations across the full dynamic range for an evaluation of each mycotoxin. Where necessary additional standards were added to improve the curve shape so that the $\mathrm{IC}_{50}$ of the curve could be determined. Cross reactivity was determined from the following calculation whereby the main mycotoxin refers to ZEA, T2 and FUM B1.

$\%$ Cross reactivity $=\mathrm{IC}_{50}$ main mycotoxin $/ \mathrm{IC}_{50}$ mycotoxin to be determined $\mathrm{x} 100$

\subsection{Intra and inter spotting precision}

Microtitre plates were spotted as described in section 3.7. Antibodies for ZEA (ABZ 1/10,000), T2 (ABT-1 1/4000) and FUM (ABF-2 1/4000) were examined during the assay. Intra-spotting precision ( $n=96$ ) was examined by analysing the mean intensity, of the maximum antibody binding achieved, on application of the 0 ppb standard in all 96 wells of the microtitre plate prepared using both PBS and methanol. Inter-spotting precision was assessed and examined over three separate spotting days. The mean intensity, of the maximum antibody binding achieved on application of the 0 ppb standard, from all 96 wells was calculated and compared 
over the three different spotting days. Intra and inter spotting precision was determined from the coefficient of variation $(\% \mathrm{CV})$.

Intra-spotting precision:

$\% \mathrm{CV}=$ Standard deviation (calculated from the intensity of $0 \mathrm{ppb}$ ) from 96 wells of one microtitre plate / Mean intensity of $0 \mathrm{ppb}$ from 96 wells of one microtitre plate $\mathrm{x} 100$

Inter-spotting precision:

$\% \mathrm{CV}=$ Standard deviation (calculated from the mean intensity of the $0 \mathrm{ppb}$ from 96 wells) of three microtitre plates / Mean intensity of 0 ppb from 96 wells of three microtitre plates x 100

\subsection{Sample preparation}

Blank and naturally incurred ground maize and wheat samples $(2.5 \mathrm{~g} \pm 0.02 \mathrm{~g})$ were weighed into plastic centrifuge tubes. Methanol/deionised water (70/30, v/v, $12.5 \mathrm{ml})$ was added and the samples were mixed for 3 min using a roller mixer. Samples were filtered (Whatman 1 filter paper) and the extracts were diluted 1 in 3 in deionised water to give a final percentage of methanol at $23.3 \%(\mathrm{v} / \mathrm{v})$. All samples were also analysed at a further dilution of 1 in 15 with sample $9 \mathrm{E}$ additionally analysed at a further dilution of 1 in 60 . This was to allow the intensity determined for highly contaminated samples to present within the calibration curve range. These further dilutions were carried out in $23.3 \%$ methanol to keep the final percentage of methanol comparable to the calibration curve.

\subsection{Matrix effects}

Microtitre plates were spotted as described in section 3.7. Antibodies for ZEA (ABZ 1/10,000), T2 (ABT-1 1/4000) and FUM (ABF-2 1/4000) were examined during the assay. Blank material 
for both maize and wheat were sourced by Queen's University Belfast and were confirmed as blank for the analytes of interest by mass spectrometry. Six calibration curves were examined including PBS, methanol, maize spiked at the start of extraction, wheat spiked at the start of extraction, maize spiked at the end of extraction and wheat spiked at the end of extraction.

\subsection{Sample analysis}

Microtitre plates were spotted as described in section 3.7. Antibodies for ZEA (ABZ 1/10,000), T2 (ABT-1 1/4000) and FUM (ABF-2 1/4000) were examined during the assay. Maize and wheat samples $(n=8)$ were extracted following the sample preparation method described in section 3.10. The sample concentrations for maize and wheat were determined from a 4 parameter fit calibration curve using BIAevaluation version 4.1 software (Biacore, GE Healthcare). Results were compared to those obtained by analysing the same samples with Tecna screening test kits (Celer ZON v3, Celer T2 and Smart Strip FUMO) and HPLC/MS. Sample MA110 was a Reference Material whose FUM concentration was assessed by Test Veritas (Padova, I).

\section{Results and Discussion}

\subsection{Optimisation}

In the optimisation of the ZEA assay it was noted that spot intensity was very low for the spotting concentration of $0.5 \mu \mathrm{g} / \mathrm{ml}$ with many spots not visible. Very little difference was observed in intensity between the four different spotting volumes $(330,670,1000,2000 \mathrm{pl})$ within each spotting concentration $(0.5,5$ and $50 \mu \mathrm{g} / \mathrm{ml})$. Nonetheless a spotting volume of $1000 \mathrm{pl}$ was selected as visually spots were easier to see for the alignment of grids.

Additionally, intensity decreased as the alkaline phosphatase anti-rabbit IgG dilution increased to $1 / 20,000$ with a dilution of $1 / 500$ chosen for the assay. A spotting concentration for ZEA of 
$50 \mu \mathrm{g} / \mathrm{ml}$ and an antibody dilution ZEA ABZ (1/10000) was therefore selected as this provided a suitable intensity and differential between negative and positive for the development of a calibration curve. For the optimisation of T2 and FUM parameters it was observed that spots were not visible for FUM antibody ABF-1 and T2 antibodies ABT-1 and ABT-2 at the spotting concentration of $10 \mu \mathrm{g} / \mathrm{ml}$. Very little difference in spot intensity was observed between 50, 100 and $200 \mu \mathrm{g} / \mathrm{ml}$ spotting concentrations for T2 and FUM. As the spotting concentration increased the sensitivity of the assay decreased and a higher antibody dilution was required. However, at the highest antibody dilutions the spots were barely visible with quite low spot intensities obtained. A spotting concentration of $50 \mu \mathrm{g} / \mathrm{ml}$ was therefore selected for $\mathrm{T} 2$ and FUM with antibody dilutions $1 / 4000$ for both ABT-1/ABT-2 and ABF-1/ABF-2 antibodies as these conditions provided suitable intensity and differential between negative and positive to allow further assay development.

\subsection{Individual calibration curves}

Individual calibration curves, in a nine spot format (3x3 array), for ZEA (ABZ antibody), T2 (ABT-1 and ABT-2 antibodies) and FUM (ABF-1 and ABF-2 antibodies) using both PBS and methanol were examined. For T2 and FUM antibodies ABT-1 and ABF-2 were selected for the final multiplex assay as illustrated in Figures 1 and 2. The average spot intensity of the nine spots per well was calculated and the average of the two wells was determined. The spot intensity for ZEA was approximately 2000 pixels $(0 \mathrm{ppb})$ and sensitivity of the assay (based on the $50 \%$ inhibition concentrations) decreased from $21.9 \mathrm{ppb}$ to $100.2 \mathrm{ppb}$ between PBS and methanol calibration curves (Table 1). Since mycotoxins such as ZEA tend to be lipophilic and organic solvents are often used to extract analytes from samples for immunoassays it is necessary to use a water miscible organic solvent in the assay buffer for ELISAs. Methanol tends to be the most widely used. However, it has been shown that with increased methanol 
concentration the sensitivity of the assay can decrease [37]. The methanol may affect either the immobilised protein conjugate or the antibody protein structure causing reduced binding to the ZEA due to interference and thereby decreasing the sensitivity. For T2 the spot intensity for both antibodies was very low at approximately $800-1000$ pixels $(0 \mathrm{ppb})$ whereby grids had to be manually aligned. Sensitivity for both the ABT-1 and ABT-2 antibodies were very similar based on the $50 \%$ inhibition concentrations $(1 \mathrm{ppb})$ and remained similar between PBS and methanol calibration curves (Table 1). The ABT-1 antibody was selected for the multiplex calibration curve as the intensity was slightly higher and whereby automatic grid alignment could be achieved to determine signal intensity. For FUM the starting intensity ( $0 \mathrm{ppb})$ for the ABF-2 antibody was slightly higher at approximately 2000 pixels compared to the ABF-1 antibody (1500 pixels). Sensitivity for both the ABF-1 and ABF-2 antibodies were very similar based on the $50 \%$ inhibition concentrations; however, sensitivity did decrease from approximately $60 \mathrm{ppb}$ to $100 \mathrm{ppb}$ between PBS and methanol calibration curves (Table 1). Again, the methanol may affect the FUM antibody and cause some interference in binding to FUM decreasing the sensitivity. The ABF-2 antibody was selected as the antibody to use for the multiplex calibration curve because the intensity was much higher. Reduced sensitivities for the methanol calibration curves was possibly because methanol will cause reduced antibody binding due to the target due to solvent effects on the antibody. However, for these mycotoxins due to their solubility properties it is necessary to use an extraction solvent in the sample preparation methods.

\subsection{Multiplex calibration curves}

Multiplex calibration curves, in a 12 spot format (4x3 array), for ZEA (ABZ antibody), T2 (ABT-1 antibody) and FUM (ABF-2 antibody) using both PBS (Figure 1) and methanol (Figure 2) over six wells and over three days are illustrated. Variation in the average intensity of the 
four spots per well was 18.8, 20.5 and $12.7 \%$ for the PBS calibration curve and 18.5, 20.1 and 12.4 for the methanol calibration curve for ZEA, T2 and FUM respectively. This was determined as the average $\% \mathrm{CV}$ of all standards in the calibration curve over 6 wells and 3 days. Improved curve shapes were produced for T2 as multiplex calibration curves with only a slight difference between PBS and methanol. Spot intensity for T2 was very low therefore the grid alignment had to be completed manually. Calibration curves for FUM were similar between PBS and methanol until the final two standards. It is, however, more difficult to align the grids when the spot intensity is faint and so this may account for this difference. Sensitivity, assessed by examining the $50 \%$ inhibition concentration of the assay, for the nanoarray for ZEA, T2 and FUM are presented in Table 1. Sensitivity decreased for ZEA in PBS from 21.9 $\mathrm{ppb}$ (individual) to $70.1 \mathrm{ppb}$ (multiplex) and similarly for methanol from $100.2 \mathrm{ppb}$ (individual) to $172.4 \mathrm{ppb}$ (multiplex). The standard deviation of the intensity of each spot is greater in the multiplex assay for ZEA indicating that there may be interference occurring from the other assays. Sensitivity decreased for T2 in PBS from $1.0 \mathrm{ppb}$ (individual) to $2.8 \mathrm{ppb}$ (multiplex) and similarly for methanol from $0.9 \mathrm{ppb}$ (individual) to $3.2 \mathrm{ppb}$ (multiplex). Similar to ZEA the standard deviation of the intensity of each spot is greater in the multiplex assay for T2 indicating that there may be interference occurring from the other assays. Finally, sensitivity also decreased for FUM in PBS from $63.9 \mathrm{ppb}$ (individual) to $90.9 \mathrm{ppb}$ (multiplex) and similarly for methanol from $105.0 \mathrm{ppb}$ (individual) to $129.3 \mathrm{ppb}$ (multiplex). Reduced sensitivities between the individual and multiplex calibration curves is possibly due to interference and non-specific binding of reagents between the assays. As the standard deviation of the spots increased more significantly in the ZEA and T2 assays when multiplexed compared to the FUM assay the reagents in these assays may be interacting. Multiplexing assays with different antibodies may lead to some degree of interference between antibodies and between 
targets even due to steric hindrance. Nonetheless, certain benefits of multiplexing outweigh the loss of sensitivity in most applications.

\subsection{Cross reactivity}

For the multiplex nanoarray the characterisation of the antibodies, especially in regard to their cross reactivity is important to know. The cross reactivity profile for each antibody was examined by analysing individual calibration curves prepared in methanol for ZEA, T2, HT2, FUM B1, FUM B2, FUM B3 and DON as illustrated in Figure 3. The results demonstrated that the T2 antibody ABT- 1 showed $74 \%$ cross reactivity to HT2. FUM antibody ABF-2 showed $48 \%$ and $24 \%$ cross reactivity to FUM B2 and FUM B3 respectively. Moreover, the antibodies used turned out to be specific for their corresponding mycotoxin and showed minimal to no cross reactivity for the concentrations used in the assay for the other mycotoxins (Figure 3).

\subsection{Intra and inter spotting precision}

The data for the spotting precision of each mycotoxin was examined by analysing a $0 \mathrm{pbb}$ standard in all 96 wells of a three-plex (ZEA, T2 and FUM) spotted system using both PBS and methanol. Results are shown in Table 2. Intra-spotting precision was 6, 11 and $10 \%$ for PBS and 5, 11 and $12 \%$ for methanol for ZEA, T2 and FUM respectively. Inter-spotting precision was 4, 14 and $6 \%$ for PBS and 3, 9 and $16 \%$ for methanol for ZEA, T2 and FUM respectively over 3 spotting days.

\subsection{Sample preparation}

Both maize and wheat were extracted according to the protocol described in section 3.11. All extracts were diluted 1 in 3 and 1 in 15 to give a final dilution of 15 and 75 and a percentage of methanol at $23.3 \%$. Sample 9E was highly contaminated for T2 and FUM and therefore the 
extract was diluted 1 in 60 to give a final dilution of 300 so that the response would present within the calibration range. Taking into account the $50 \%$ inhibition concentration and the 1 in 3 extraction dilution this converts to $1050.9,42.0$ and $1364.1 \mu \mathrm{g} / \mathrm{kg}$ for ZEA, T2 and FUM respectively which meets the regulatory limits for these mycotoxins in feed. It was observed that for wheat samples using only a 1 in 3 dilution produced a white cloudy extract. There is some component of the wheat that is causing a cloudy supernatant which is not present in the maize. It may therefore be better to increase the dilution to 1 in 15 for the assay (final dilution 75). This would dilute out the extract and minimize the cloudy extract interfering with the test.

\subsection{Matrix effects}

The calibration curves were assessed for ZEA (ABZ antibody), T2 (ABT-1 antibody) and FUM (ABF-2 antibody) for matrix effects in maize (Figure 4) and wheat (Figure 5). . For ZEA the intensity for the $0 \mathrm{ppb}$ standard for maize and wheat were very similar to the PBS and methanol curves. However, the difference in the curve shape was more pronounced for the maize and wheat. Sensitivity for ZEA in matrix is somewhat reduced for both maize and wheat (Table 1). For T2 the intensity for the $0 \mathrm{ppb}$ standard for maize and wheat was approximately $16 \%$ and $13 \%$ lower than the PBS and methanol curves respectively. The calibration curves for maize showed a large drop between the first two standards giving a poor curve shape. In comparison, the wheat produced a better curve shape and compared better with the PBS and methanol curves. Sensitivity for T2 for both maize and wheat is reduced when spiked at the start of the extraction, however, is similar to PBS and methanol curves when spiked at the end into negative extract (Table 1). For FUM the intensity for the 0 ppb standard for maize was approximately $11 \%$ lower than the PBS and methanol curves with wheat showing an intensity approximately $7 \%$ higher in comparison. The calibration curve for the maize spiked at the end of the extraction showed a slightly different curve shape compared to the others. Sensitivity for 
FUM in matrix is somewhat reduced for both maize and wheat (Table 1). A differential in the calibration curves prepared pre and post extraction illustrates the extent of the recovery of the assay. It was evident that due to substantial differences between these two curves that recovery was not $100 \%$ and whereby some mycotoxins may not be extracted during the extraction process. This would mean that the concentration of unknown samples should be determined from a calibration curve prepared pre-extraction during the final validation of the assay or the extraction procedure should be examined further to help improve the recovery of the assay.

\subsection{Sample analysis}

The analysis of the wheat and maize samples $(n=8)$ using the multiplex nanoarray in comparison with other screening methods and HPLC are presented in Table 3. Comparing the nanoarray results to concentrations for ELISA, LFD and HPLC/MS it was observed that for ZEA there were five samples that correlate in terms of negative and positive results. Samples L2 and GT were both expected to be $<50 \mathrm{ppb}$ but have a higher concentration when analysed by the mycotoxin nanoarray at $132 \mathrm{ppb}$ and $206 \mathrm{ppb}$ respectively. Additionally, sample MA110 had a higher concentration when analysed by the mycotoxin nanoarray. For the ZEA assay only ZEA was determined whereby for future work other analogues of ZEA should be examined to ensure differences between the assays are not due to the cross-reactivity towards these analogues. For T2 there were six samples that correlate in terms of negative and positive results. Sample GT was expected to be $<5 \mathrm{ppb}$ but had a higher concentration of $86 \mathrm{ppb}$ when analysed by the mycotoxin nanoarray. Additionally, sample MA110 had a higher concentration when analysed by the mycotoxin nanoarray. Finally, for FUM there were seven samples that match up in terms of negative and positive results. The reference material MA110 had a higher concentration of $116 \mathrm{ppb}$ when analysed by the mycotoxin nanoarray compared to the certified concentration of $<50 \mathrm{ppb}$. Differences detected for certain samples may be due to the non- 
homogeneity of the sample as it is well known that mycotoxins are very heterogeneous in nature [38], sensitivities of the different testing platforms and possible variations in cross reactivity profiles of the antibodies used to analogues of the compounds in the screening tests as analysed in different laboratories at different times. The sample for analysis provided for the nanoarray study was relatively small and in this study the analysis by the different methods was performed over different sites and whereby the sample may have been stored under different conditions. The differences detected for certain samples may also be due to the heterogeneity in distribution of the mycotoxin in the sample provided to the different sites. It is well established that mycotoxin distribution in a sample can be very heterogeneous in nature and storage conditions are important. This paper is a proof of concept and feasibility study to show that the mycotoxin nanoarray application is highly feasible. Further work would include a single laboratory validation and substantive survey of real samples in direct analytical comparison with other routine testing platforms.

\subsection{Image and Data Analysis}

For the implementation of the nanoarray application the reader must be employed that can record the spot intensity at the spatially defined zones compared to the whole well as in current laboratory ELISA applications. For novice end users a current major drawback of the reader technology is the time required for image analysis and data processing. Currently all 96 wells are imaged and each image must be opened by the software for grid alignment and calculation of spot intensity. Aligning grids for one microtitre plate takes approximately $2 \mathrm{hr}$, generating 96 excel sheets. Further data processing can take a further $1 \mathrm{hr}$ therefore data processing per plate can take up to $3 \mathrm{hr}$. This is not applicable from a commercial point and unless data processing software solutions can be addressed it would be unsuitable for commercial and routine laboratory use. Reference spots should be assessed and included in future work which 
may help with grid alignment. For this to be a viable method the reader software needs to be enhanced for automated data processing for all 96 wells generating calibration curves and interpolating unknown samples to these curves. Additionally, another drawback is that any dust or particles present in the well can interfere with the image and spot intensity especially if it is covering a spot within the nanoarray. Therefore, clean room facilities for production and use may need to be a consideration.

\section{Conclusions}

Monitoring programmes for mycotoxins have become a necessity because of the potential consequences to human and animal health. New innovations in technology applications to improve surveillance for the industry are essential. A sensitive and novel multiplex nanoarray has been developed allowing for the semi-quantitative and simultaneous screening of three regulated mycotoxins. The immunological format offers a high throughput detection method for ZEA, T2 and FUM, whereby with improved software utilities on the reader results, results would be available in around $70 \mathrm{~min}$. The benefit to this system is that it follows established ELISA protocols whereby laboratories with immunological screening methods already in place have end users familiar to the steps in analysis and comparable to LFD offers a higher throughput of samples. The simplicity, sensitivity and comparative labour to ELISA of the mycotoxin nanoarray assay means it could be used as a screening method in a monitoring environment for the presence of these harmful mycotoxins in food. This technology demonstrates the potential feasibility for an easy to use and sensitive multiplex mycotoxin detection method. This study has shown some very promising data and required considerations that are worthy of further research to determine the nanoarray suitability for a commercial diagnostic test. Prior to implementation a full validation and inter-laboratory trial of the nanoarray should be conducted following accreditation guidelines for screening methods. 


\section{Acknowledgements}

This study was funded by Regione Friuli Venezia Giulia (I): "Legge Regionale 47/78" and by the Advanced ASSET project, partly funded through InvestNI and from the European Sustainable Programme 2007-2013 under the European Regional Development Fund (“ERDF”).

\section{References}

[1] P. Steyn, Mycotoxins, general view, chemistry and structure, Toxicol. Lett. 82-3 (1995) 843-851.

[2] M.E. Zain, Impact of mycotoxins on humans and animals, J. Saudi Chem. Soc. 15 (2011) $129-144$.

[3] L. Pinotti, M. Ottoboni, C. Giromini, V. Dell'Orto, F. Cheli, Mycotoxin Contamination in the EU Feed Supply Chain: A Focus on Cereal Byproducts, Toxins. 8 (2016) 45. [4] R. Chauhan, J. Singh, T. Sachdev, T. Basu, B.D. Malhotra, Recent advances in mycotoxins detection, Biosens. Bioelectron. 81 (2016) 532-45.

[5] H.P. van Egmond, R.C. Schothorst, M.A. Jonker, Regulations relating to mycotoxins in food, Anal. Bioanal. Chem. 389 (2007) 147-157.

[6] K. Nesic, S. Ivanovic, V. Nesic, Fusarial Toxins: Secondary Metabolites of Fusarium Fungi, Rev. Environ. Contam. T. 228 (2014) 101-120.

[7] L. Anfossi, C. Baggiani, C. Giovannoli, G. D'Arco, G. Giraudi, Lateral-flow immunoassays for mycotoxins and phycotoxins: a review, Anal. Bioanal. Chem. 405 (2013) 467-480. 
[8] J. D'Mello, C. Placinta, A. Macdonald, Fusarium mycotoxins: a review of global implications for animal health, welfare and productivity, Anim. Feed Sci. Technol. 80 (1999) 183-205.

[9] D.R. Milicevic, M. Skrinjar, T. Baltic, Real and Perceived Risks for Mycotoxin Contamination in Foods and Feeds: Challenges for Food Safety Control, Toxins. 2 (2010) $572-592$.

[10] L. Anfossi, C. Giovannoli, C. Baggiani, Mycotoxin detection, Curr. Opin. Biotechnol. 37 (2016) 120-126.

[11] Commission regulation (EC) No 1881/2006 of 19 December 2006 setting maximum levels for certain contaminants in foodstuffs. Off. J. Eur. Union (2006) L364, 5-24. [12] Commission regulation (EC) No 1126/2007 of 28 September 2007 setting maximum levels for certain contaminants in foodstuffs as regards Fusarium toxins in maize and maize products. Off. J. Eur. Union (2007) L255, 14-17.

[13] Commission recommendation of 27 March 2013 on the presence of T-2 and HT-2 toxin in cereals and cereal products. Off. J. Eur. Union (2013) L91, 12-15.

[14] Commission recommendation of 17 August 2006 on the presence of deoxynivalenol, zearalenone, ochratoxin A, T-2 and HT-2 and fumonisins in products intended for animal feeding. Off. J. Eur. Union (2006) L229, 7-9.

[15] C.M. Maragos, Biosensors for mycotoxin analysis: recent developments and future prospects, World Mycotoxin Journal. 2 (2009) 221-238.

[16] B. Huybrechts, E.K. Tangni, P. Debongnie, J. Geys, A. Callebaut, A review of analytical methods for determining mycotoxins in agricultural products, Cahiers Agricultures. 22 (2013) $202-215$

[17] V.G. Amelin, N.M. Karaseva, A.V. Tret'yakov, Chromatographic methods for the determination of mycotoxins in food products, J. Anal. Chem. 68 (2013) 195-205. 
[18] J. O'Mahony, L. Clarke, M. Whelan, R. O'Kennedy, S.J. Lehotay, M. Danaher, The use of ultra-high pressure liquid chromatography with tandem mass spectrometric detection in the analysis of agrochemical residues and mycotoxins in food - Challenges and applications, J. Chrom. A. 1292 (2013) 83-95.

[19] V.L. Pereira, J.O. Fernandes, S.C. Cunha, Mycotoxins in cereals and related foodstuffs: A review on occurrence and recent methods of analysis, Trends Food Sci. Technol. 36 (2014) 96-136.

[20] A.T. Aberg, A. Solyakov, U. Bondesson, Development and in-house validation of an LC-MS/MS method for the quantification of the mycotoxins deoxynivalenol, zearalenone, T2 and HT-2 toxin, ochratoxin A and fumonisin B1 and B2 in vegetable animal feed, Food Addit. Contam. 30 (2013) 541-549.

[21] E. Ates, K. Mittendorf, J. Stroka, H. Senyuva, Determination of fusarium mycotoxins in wheat, maize and animal feed using on-line clean-up with high resolution mass spectrometry, Food Addit. Contam. 30 (2013) 156-165.

[22] Y.Y. Tang, H.Y. Lin, Y.C. Chen, W.T. Su, S.C. Wang, L.C. Chiueh, Y.C. Shin, Development of a Quantitative Multi-Mycotoxin Method in Rice, Maize, Wheat and Peanut Using UPLC-MS/MS, Food Anal. Methods. 6 (2013) 727-736.

[23] V.M.T. Lattanzio, B. Ciasca, S. Powers, A. Visconti, Improved method for the simultaneous determination of aflatoxins, ochratoxin A and Fusarium toxins in cereals and derived products by liquid chromatography-tandem mass spectrometry after multi-toxin immunoaffinity clean up, J. Chromatogr. A. 1354 (2014) 139-143.

[24] P.J. Fernandes, N. Barros, J.L. Santo, J.S. Camara, High-Throughput Analytical Strategy Based on Modified QuEChERS Extraction and Dispersive Solid-Phase Extraction Clean-up Followed by Liquid Chromatography-Triple-Quadrupole Tandem Mass Spectrometry for Quantification of Multiclass Mycotoxins in Cereals, Food Anal. Methods. 8 (2015) 841-856. 
[25] Y. Huang, Y. Xu, Q. He, J. Chu, B. Du, J. Liu, Determination of zearalenone in corn based on a biotin-avidin amplified enzyme-linked immunosorbent assay, Food Agric. Immunol. 25 (2014) 186-199.

[26] Y. Li, X. Luo, S. Yang, X. Cao, Z. Wang, W. Shi, S. Zhang, High Specific Monoclonal Antibody Production and Development of an ELISA Method for Monitoring T-2 Toxin in Rice, J. Agric. Food Chem. 62 (2014) 1492-1497.

[27] A.E. Urusov, A.V. Zherdev, A.V. Petrakova, E.G. Sadykhov, O.V. Koroleva, B.B. Dzantiev, Rapid Multiple Immunoenzyme Assay of Mycotoxins, Toxins. 7 (2015) 238-254. [28] H.O. Arola, A. Tullila, H. Kiljunen, K. Campbell, H. Siitari, T.K. Nevanen, Specific Noncompetitive Immunoassay for HT-2 Mycotoxin Detection, Anal. Chem. 88 (2016) 24462452.

[29] X.C. Wang, M. Bao, F.H. Li, H.X. Fan, H.S. Li, Y. Li, S.B. Feng, J.J. Wu, Development of a sensitive, competitive, indirect ELISA for the detection of fumonisin B-1 in corn originating from Anhui province, China, J. Environ. Sci. Heal. B. 51 (2016) 107-112. [30] A. Molinelli, K. Grossalber, M. Fuehrer, S. Baumgartner, M. Sulyok, R. Krska, Development of qualitative and semiquantitative immunoassay-based rapid strip tests for the detection of T-2 toxin in wheat and oat, J. Agric. Food Chem. 56 (2008) 2589-2594.

[31] A. Molinelli, K. Grossalber, R. Krska, A rapid lateral flow test for the determination of total type B fumonisins in maize, Anal. Bioanal. Chem. 395 (2009) 1309-1316.

[32] V.M.T. Lattanzio, N. Nivarlet, V. Lippolis, S. Della Gatta, A. Huet, P. Delahaut, B. Granier, A. Visconti, Multiplex dipstick immunoassay for semi-quantitative determination of Fusarium mycotoxins in cereals, Anal. Chim. Acta. 718 (2012) 99-108.

[33] V.M.T. Lattanzio, C. von Holst, A. Visconti, Collaborative study for evaluating performances of a multiplex dipstick immunoassay for Fusarium mycotoxin screening in wheat and maize, Quality Assurance and Safety of Crops \& Foods. 6 (2014) 299-307. 
[34] V.M.T. Lattanzio, C. von Holst, A. Visconti, Experimental design for in-house validation of a screening immunoassay kit. The case of a multiplex dipstick for Fusarium mycotoxins in cereals, Anal. Bioanal. Chem. 405 (2013) 7773-7782.

[35] S. Oswald, X.Y.Z. Karsunke, R. Dietrich, E. Maertlbauer, R. Niessner, D. Knopp, Automated regenerable microarray-based immunoassay for rapid parallel quantification of mycotoxins in cereals, Anal. Bioanal. Chem. 405 (2013) 6405-6415.

[36] A. Beizaei, S.L. O'Kane, A. Kamkar, A. Misaghi, G. Henehan, D.J. Cahill, Highly sensitive toxin microarray assay for aflatoxin B1 detection in cereals, Food Control. 57 (2015) 210-215.

[37] C. Wang, Z. Wang, W. Jiang, T. Mi, J. Shen. A monoclonal antibody based ELISA for multiresidue determination of avermectins in milk. Molecules 17 (2012) 7401-7414.

[38] R. Krska, E. Welzig, F. Berthiller, A. Molinelli, B. Mizaikoff, Advances in the analysis of mycotoxins and its quality assurance. Food Addit. Contam. 22 (2005) 345-353. 
Table 1: Sensitivity of the mycotoxin nanoarray for ZEA, T2 and FUM as both individual and multiplex curves in PBS, $23.3 \%$ methanol and extract (maize and wheat) based on $50 \%$ inhibition concentrations.

\begin{tabular}{|c|c|c|c|c|c|}
\hline Mycotoxin & System & Antibody & Matrix & $\mathbf{n}$ & $\begin{array}{c}50 \% \text { Inhibition } \\
(\text { ppb) }\end{array}$ \\
\hline \multirow{8}{*}{ ZEA } & Individual & $\mathrm{ABZ}$ & PBS & 2 & 21.9 \\
\hline & Individual & $\mathrm{ABZ}$ & $23.3 \%$ methanol & 2 & 100.2 \\
\hline & Multiplex & $\mathrm{ABZ}$ & PBS & 20 & 70.1 \\
\hline & Multiplex & $\mathrm{ABZ}$ & $23.3 \%$ methanol & 20 & 172.4 \\
\hline & Multiplex & $\mathrm{ABZ}$ & Wheat spiked at start & 2 & 299.6 \\
\hline & Multiplex & $\mathrm{ABZ}$ & Wheat spiked at end & 2 & 197.4 \\
\hline & Multiplex & $\mathrm{ABZ}$ & Maize spiked at start & 2 & $>500$ \\
\hline & Multiplex & $\mathrm{ABZ}$ & Maize spiked at end & 2 & 43.6 \\
\hline \multirow{10}{*}{$\mathbf{T 2}$} & Individual & ABT-1 & PBS & 2 & 1.1 \\
\hline & Individual & ABT-1 & $23.3 \%$ methanol & 2 & 1.7 \\
\hline & Individual & ABT-2 & PBS & 2 & 1.0 \\
\hline & Individual & ABT-2 & $23.3 \%$ methanol & 2 & 0.9 \\
\hline & Multiplex & ABT-1 & PBS & 20 & 2.8 \\
\hline & Multiplex & ABT-1 & $23.3 \%$ methanol & 20 & 3.2 \\
\hline & Multiplex & ABT-1 & Wheat spiked at start & 2 & 6.0 \\
\hline & Multiplex & ABT-1 & Wheat spiked at end & 2 & 0.7 \\
\hline & Multiplex & ABT-1 & Maize spiked at start & 2 & 2.9 \\
\hline & Multiplex & ABT-1 & Maize spiked at end & 2 & 0.5 \\
\hline \multirow{10}{*}{ FUM } & Individual & ABF-1 & PBS & 2 & 61.5 \\
\hline & Individual & $\mathrm{ABF}-1$ & $23.3 \%$ methanol & 2 & 110.0 \\
\hline & Individual & $\mathrm{ABF}-2$ & PBS & 2 & 63.9 \\
\hline & Individual & $\mathrm{ABF}-2$ & $23.3 \%$ methanol & 2 & 105.0 \\
\hline & Multiplex & $\mathrm{ABF}-2$ & PBS & 20 & 90.9 \\
\hline & Multiplex & $\mathrm{ABF}-2$ & $23.3 \%$ methanol & 20 & 129.3 \\
\hline & Multiplex & $\mathrm{ABF}-2$ & Wheat spiked at start & 2 & 369.7 \\
\hline & Multiplex & $\mathrm{ABF}-2$ & Wheat spiked at end & 2 & 216.7 \\
\hline & Multiplex & $\mathrm{ABF}-2$ & Maize spiked at start & 2 & 141.7 \\
\hline & Multiplex & $\mathrm{ABF}-2$ & Maize spiked at end & 2 & 25.9 \\
\hline
\end{tabular}

Table 2: Intra and inter assay precision for the multiplex mycotoxin nanoarray for both PBS and $23.3 \%$ methanol.

\begin{tabular}{|c|c|c|c|c|c|c|}
\hline \multirow[b]{2}{*}{ Mycotoxin } & \multirow[b]{2}{*}{ Matrix } & \multicolumn{4}{|c|}{ Intra Assay Precision (\% CV) } & \multirow{2}{*}{$\begin{array}{c}\text { Inter Assay } \\
\text { Precision } \\
(\% \text { CV }) \\
(n=3)\end{array}$} \\
\hline & & $\begin{array}{c}\text { Day } 1 \\
(n=96)\end{array}$ & $\begin{array}{c}\text { Day } 2 \\
(n=96)\end{array}$ & $\begin{array}{c}\text { Day } 3 \\
(n=96)\end{array}$ & $\begin{array}{c}\text { Average } \\
(\mathbf{n}=3)\end{array}$ & \\
\hline \multirow[t]{2}{*}{ ZEA } & PBS & 6 & 5 & 6 & 6 & 4 \\
\hline & $23.3 \%$ methanol & 5 & 6 & 5 & 5 & 3 \\
\hline \multirow[t]{2}{*}{$\mathbf{T 2}$} & PBS & 15 & 7 & 11 & 11 & 14 \\
\hline & $23.3 \%$ methanol & 9 & 14 & 10 & 11 & 9 \\
\hline \multirow[t]{2}{*}{ FUM } & PBS & 9 & 8 & 13 & 10 & 6 \\
\hline & $23.3 \%$ methanol & 8 & 18 & 9 & 12 & 16 \\
\hline
\end{tabular}


Table 3: Mycotoxin concentrations for maize $(n=5)$ and wheat $(n=3)$ samples expressed as ppb. Samples were analysed by the multiplex mycotoxin nanoarray and compared to screening (ELISA and LFD) and HPLC/MS results for these mycotoxins.

\begin{tabular}{|c|c|c|c|c|c|c|c|c|c|c|c|}
\hline \multirow{2}{*}{$\begin{array}{c}\text { Sample } \\
\text { No. }\end{array}$} & \multirow{2}{*}{$\begin{array}{c}\text { Sample } \\
\text { Type }\end{array}$} & \multirow{2}{*}{$\begin{array}{c}\text { Sample } \\
\text { Code }\end{array}$} & \multicolumn{3}{|c|}{ ZEA Concentration (ppb) } & \multicolumn{3}{|c|}{ T2 Concentration (ppb) } & \multicolumn{3}{|c|}{ FUM Concentration (ppb) } \\
\hline & & & $\mathbf{E L I S A}^{\mathrm{a}}$ & HPLC & $\begin{array}{l}\text { Mycotoxin } \\
\text { Nanoarray }\end{array}$ & $\mathbf{E L I S A}^{b}$ & HPLC & $\begin{array}{l}\text { Mycotoxin } \\
\text { Nanoarray }\end{array}$ & $\mathbf{L F D}^{\mathbf{c}}$ & HPLC & $\begin{array}{l}\text { Mycotoxin } \\
\text { Nanoarray }\end{array}$ \\
\hline 1 & Maize & $9 \mathbf{E}$ & 277 & 325 & 210 & 650 & 787 & 487 & - & 37,100 & 41,756 \\
\hline 2 & Maize & $\mathbf{A Z F}$ & $<25$ & $<50$ & 17 & $<25$ & - & 0 & $<150$ & $<100$ & 0 \\
\hline 3 & Maize & $\mathbf{L} 2$ & $<25$ & - & 132 & $<25$ & $<25$ & 5 & - & 3554 & 1536 \\
\hline 4 & Maize & GR2 & 55 & 56 & 217 & $<25$ & $<25$ & 11 & 5361 & - & 2376 \\
\hline 5 & Maize & MA110* & $<25$ & $<50$ & 178 & - & $<25$ & 50 & $<150$ & $<50 *$ & 116 \\
\hline 6 & Wheat & WH43 & 223 & - & 197 & 133 & 101 & 111 & 2186 & - & 2249 \\
\hline 7 & Wheat & FR2 & $<25$ & $<5$ & 22 & $<25$ & - & 23 & - & 111 & 274 \\
\hline 8 & Wheat & GT & $<25$ & $<10$ & 206 & - & $<5$ & 86 & - & 97 & 299 \\
\hline
\end{tabular}

a Tecna Celer ZON v3

b Tecna Celer T2

c Tecna Smart Strip FUMO

*Test Veritas (I) Reference Material for fumonisins. Assigned value according to the Certificate of the Provider

- No result available 
Figure 1: Individual and multiplex calibration curves in PBS buffer for ZEA (a), T2 (b) and FUM (c) using the mycotoxin nanoarray (Individual curves: $\mathrm{n}=2$ analysis, 9 spots per analysis; Multiplex curves: $\mathrm{n}=6$ analysis, 4 spots per analysis, $\mathrm{x} 3$ spotting days).

(a) ZEA

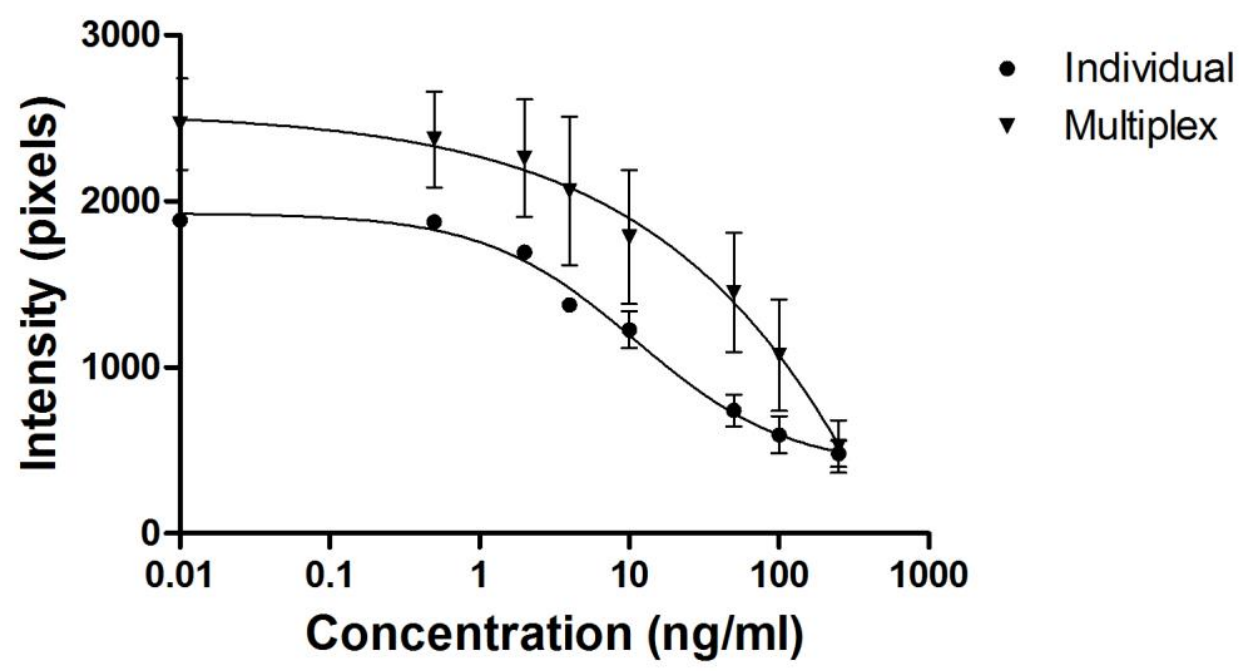

(b) T2

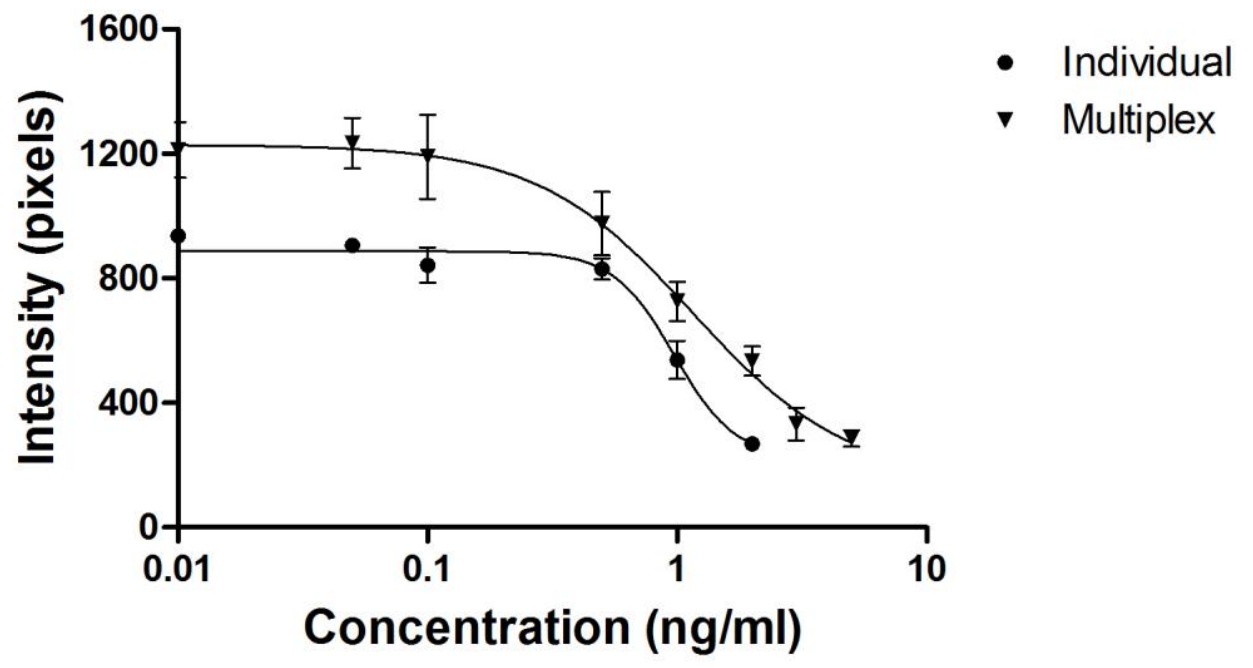


(c) FUM

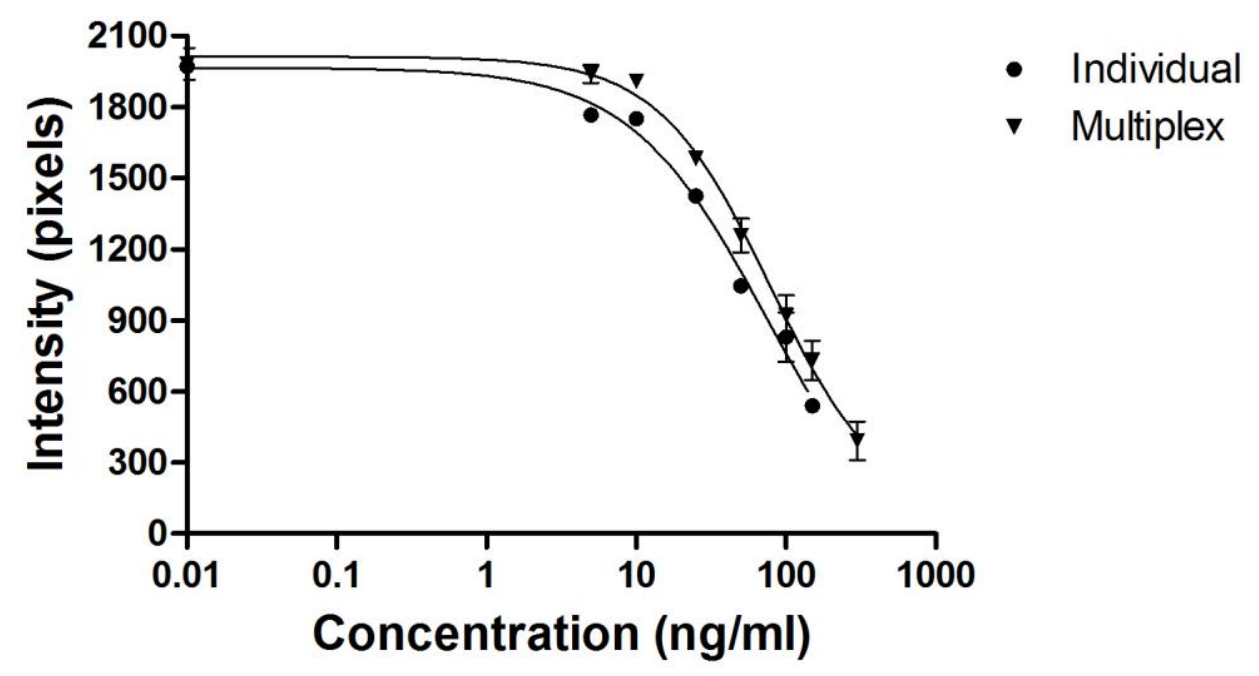


Figure 2: Individual and multiplex calibration curves in $23.3 \%$ methanol for ZEA (a), T2 (b) and FUM (c) using the mycotoxin nanoarray (Individual curves: $\mathrm{n}=2$ analysis, 9 spots per analysis; Multiplex curves: $\mathrm{n}=6$ analysis, 4 spots per analysis, $\mathrm{x} 3$ spotting days).

(a) ZEA

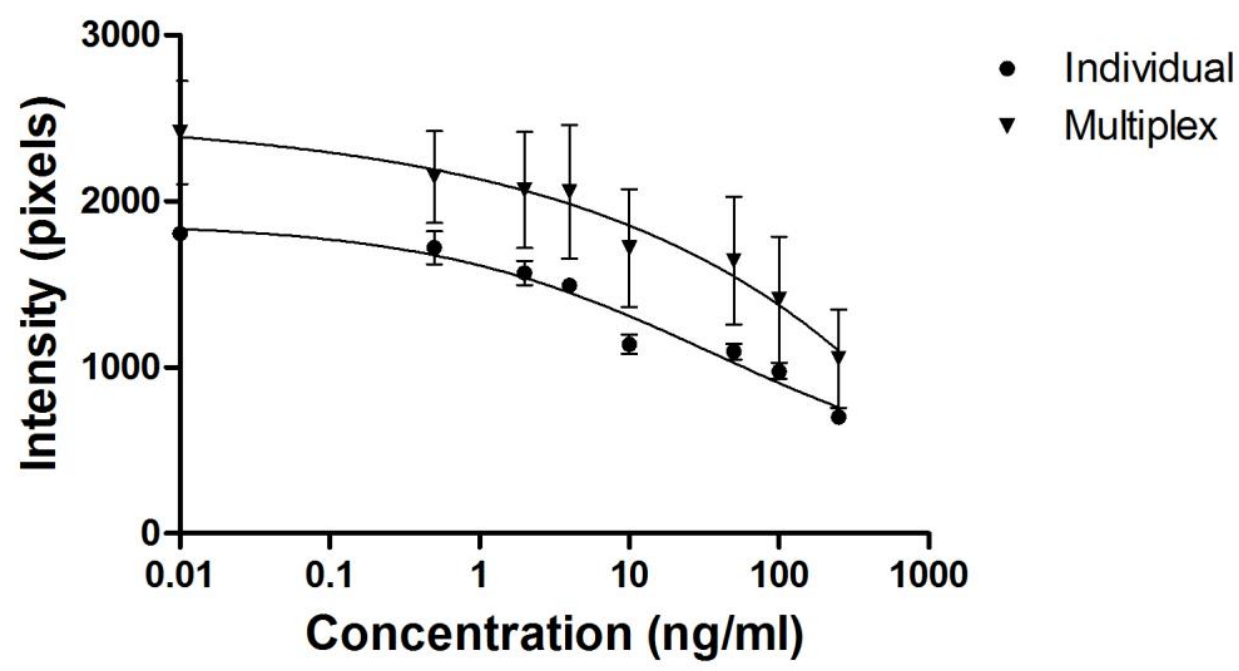

(b) T2

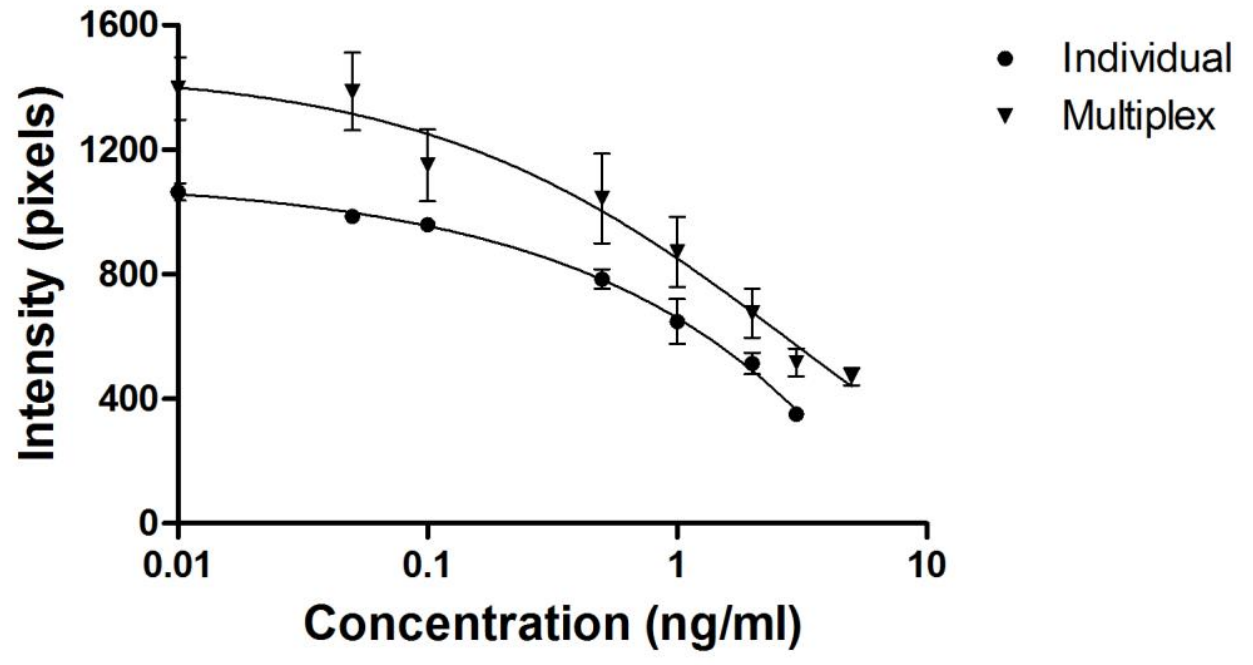


(c) FUM

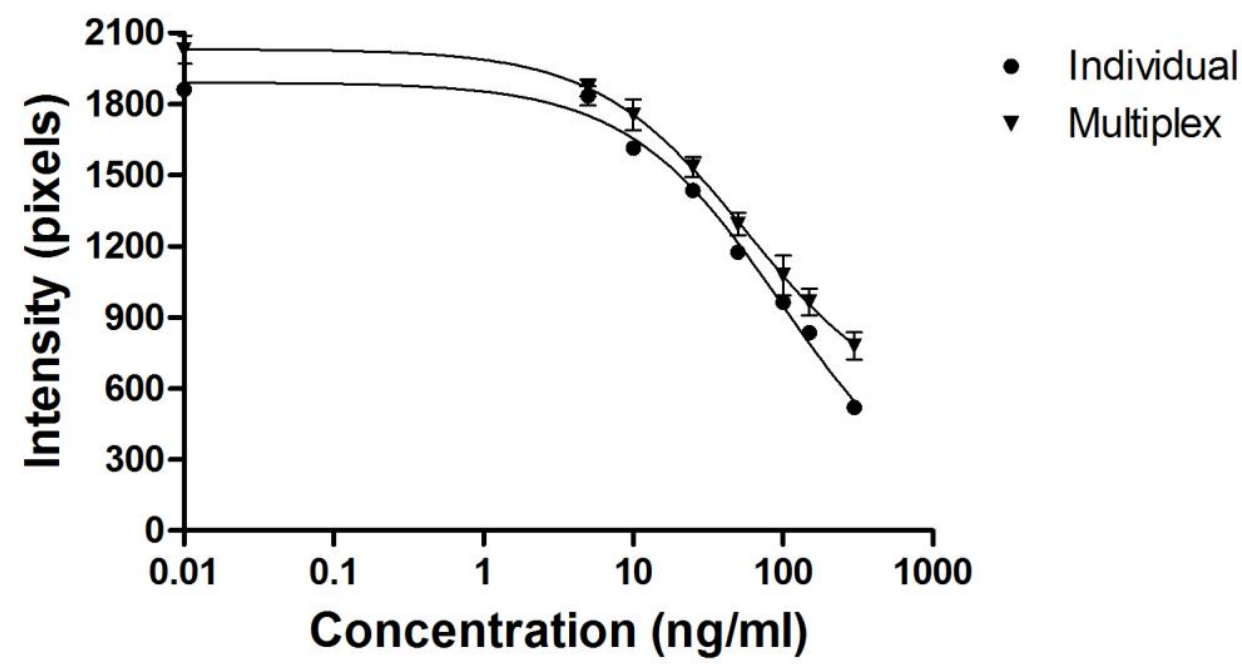


Figure 3: Cross reactivity for ZEA (a), T2 (b) and FUM (c) using the multiplex mycotoxin nanoarray ( $\mathrm{n}=2$ analysis, 4 spots per analysis).

(a) ZEA

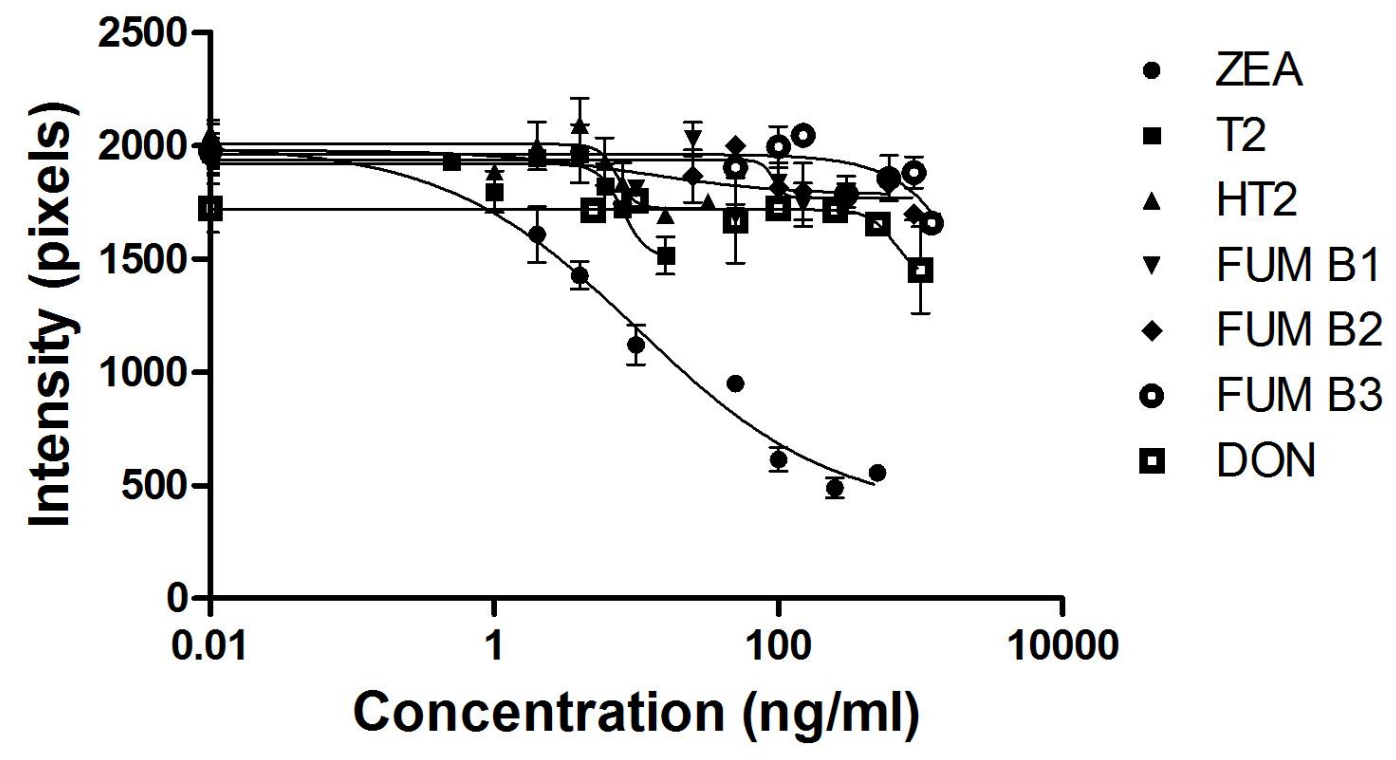

(b) T2

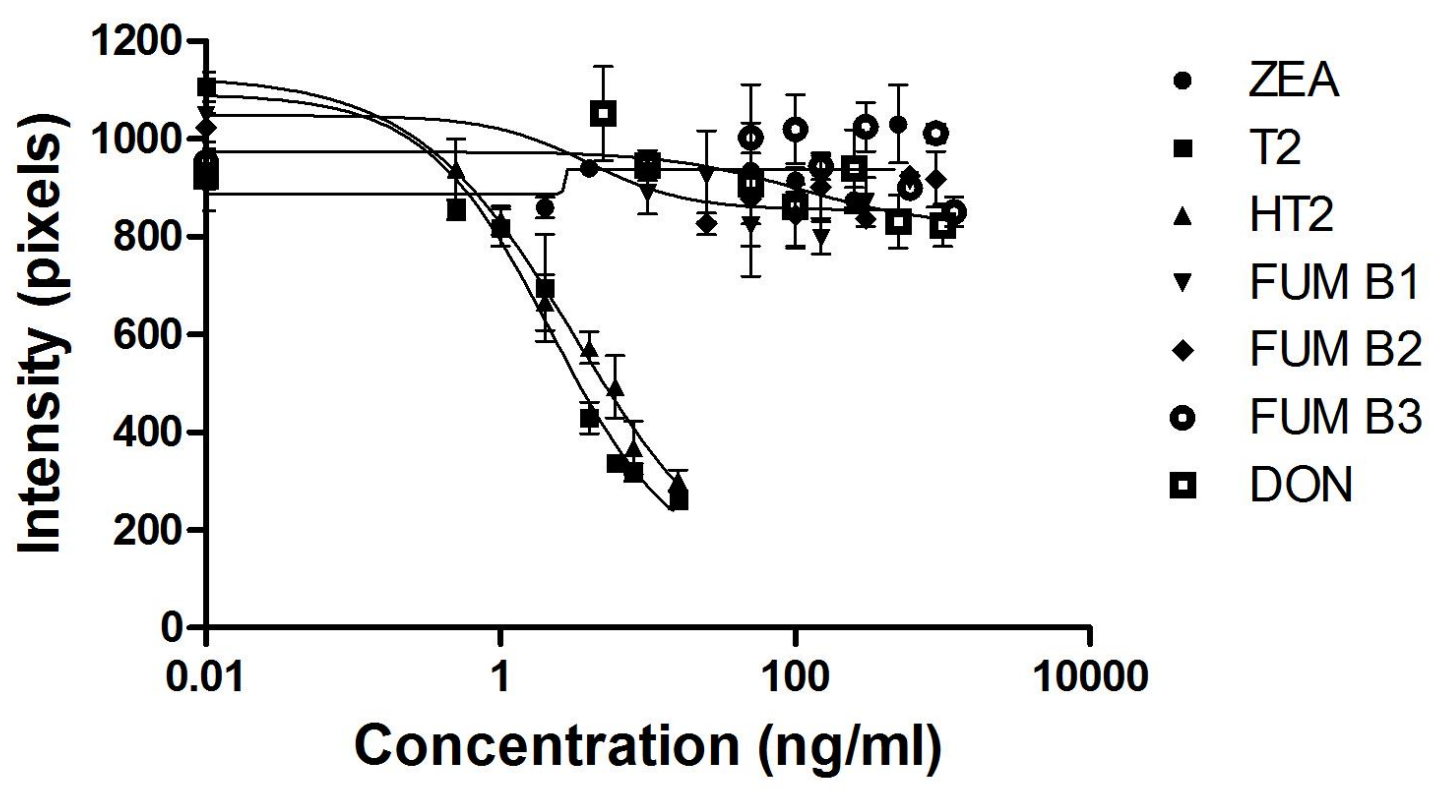


(c) FUM

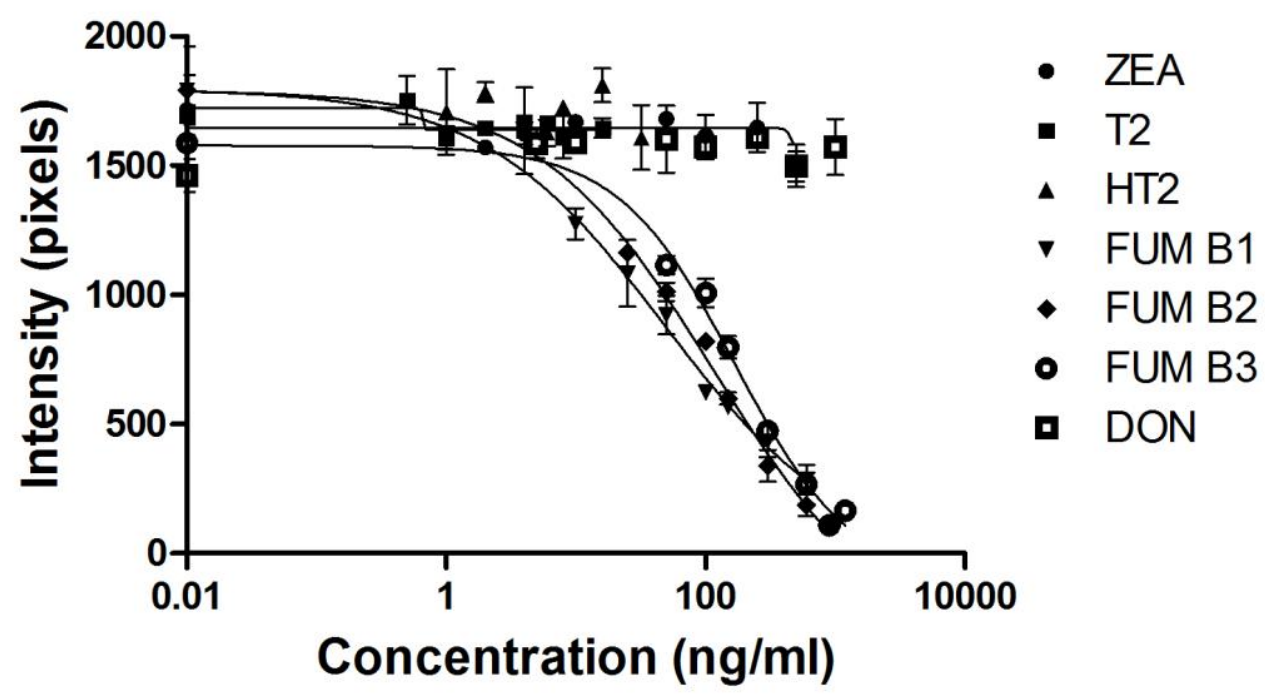


Figure 4: Matrix effects for maize for ZEA (a), T2 (b) and FUM (c) using the multiplex mycotoxin nanoarray ( $\mathrm{n}=2$ analysis, 4 spots per anaysis).

(a) ZEA

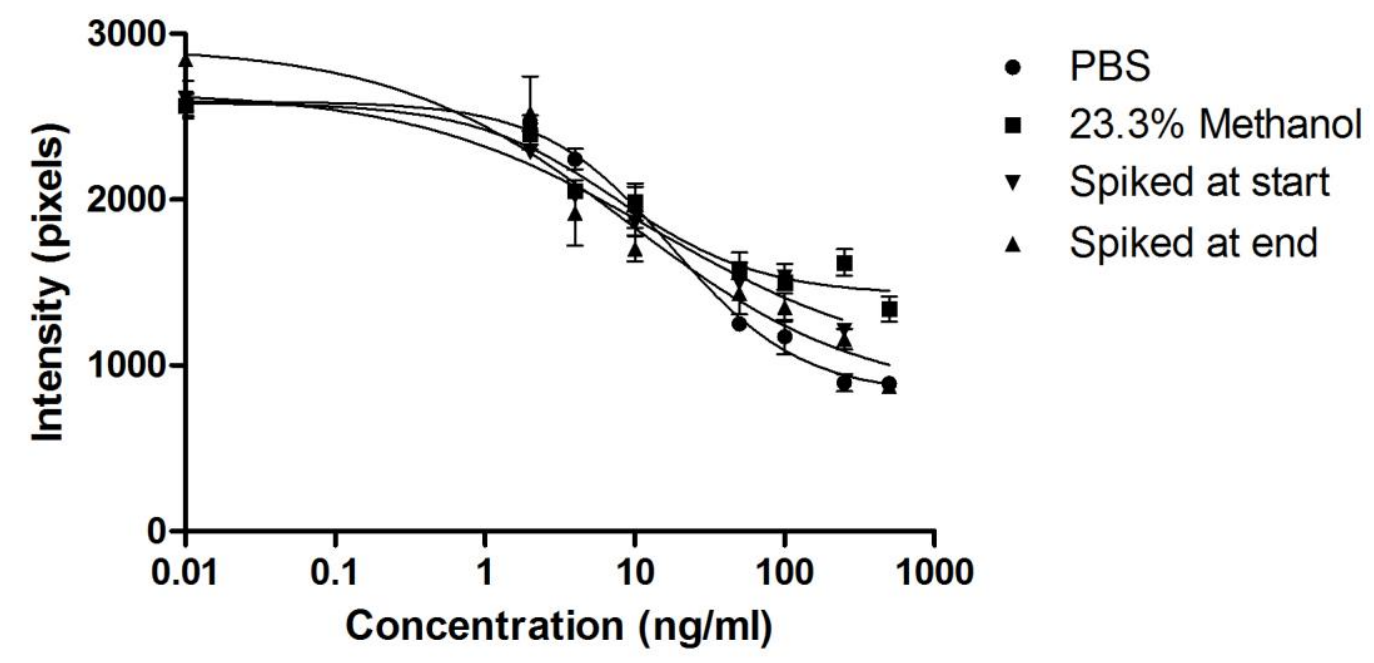

(b) T2

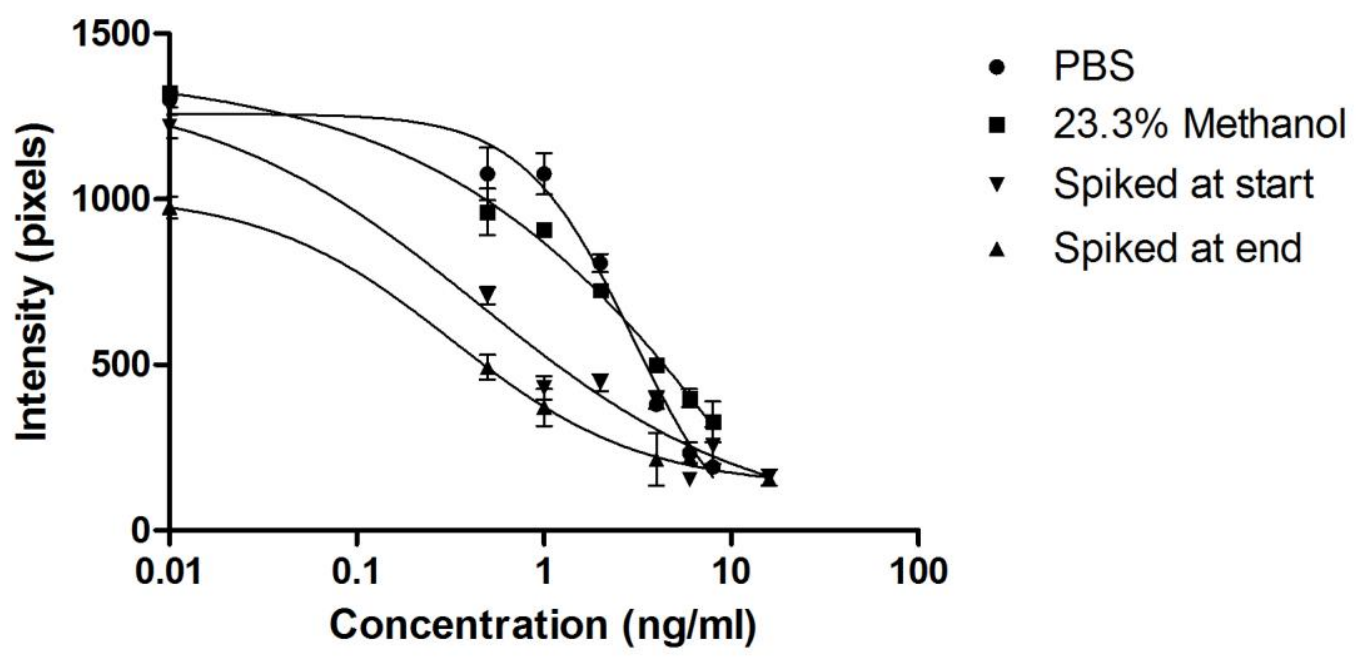


(c) FUM

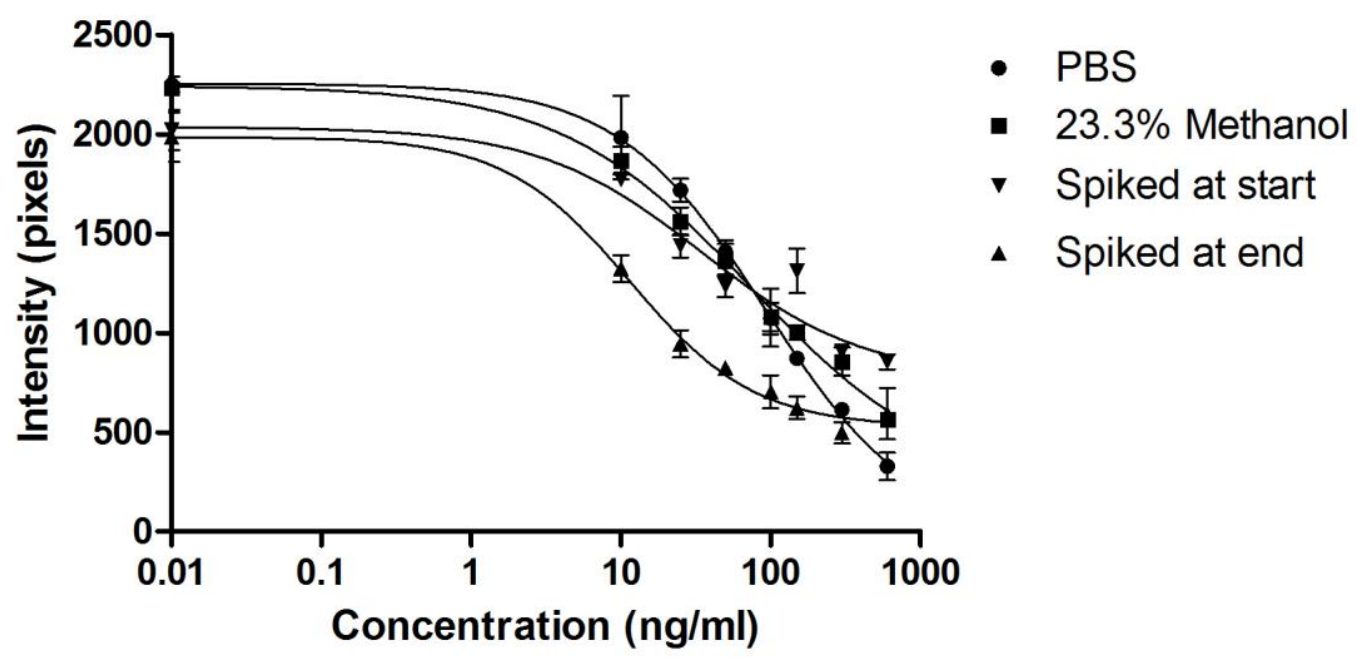


Figure 5: Matrix effects for wheat for ZEA (a), T2 (b) and FUM (c) using the multiplex mycotoxin nanoarray ( $\mathrm{n}=2$ analysis, 4 spots per anaysis).

(a) ZEA

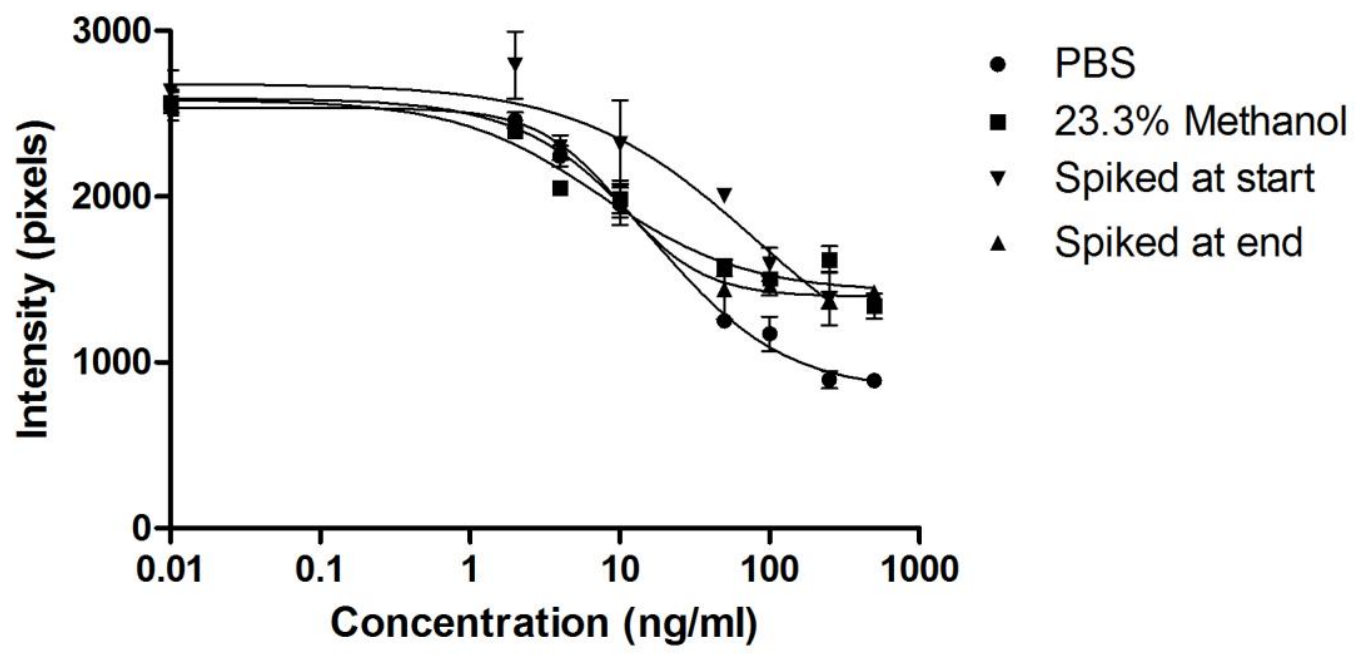

(b) T2

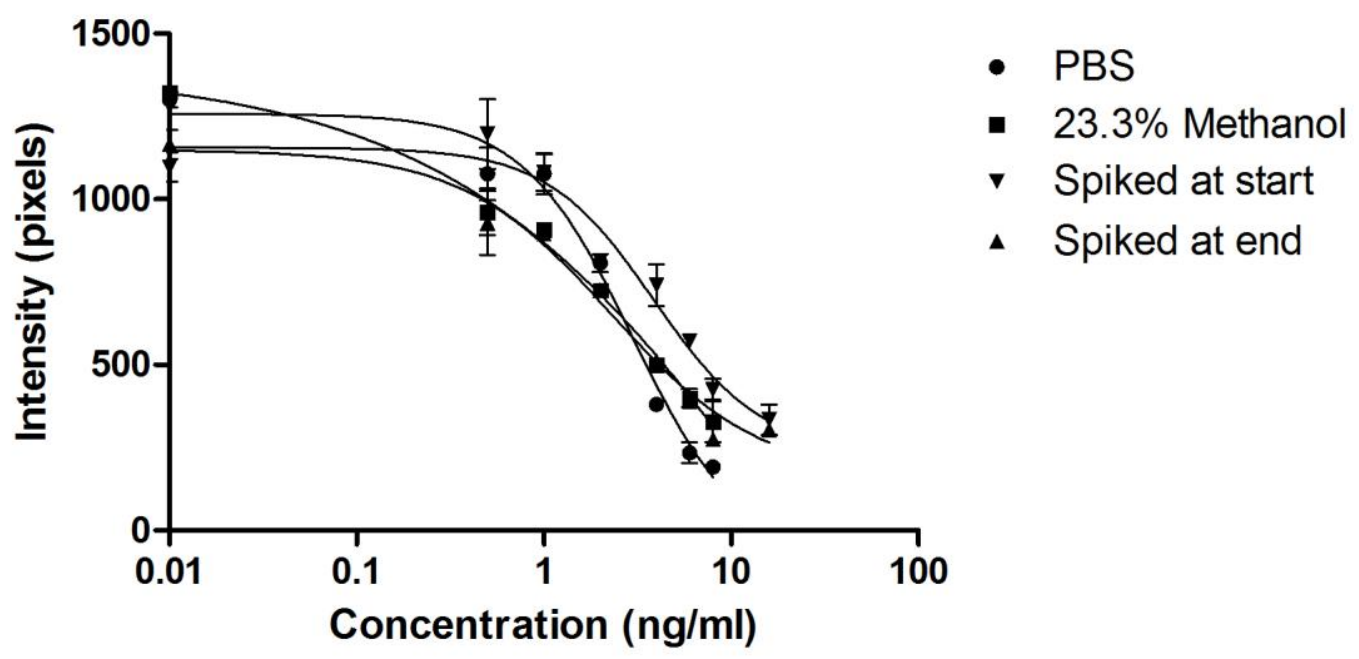


(c) FUM

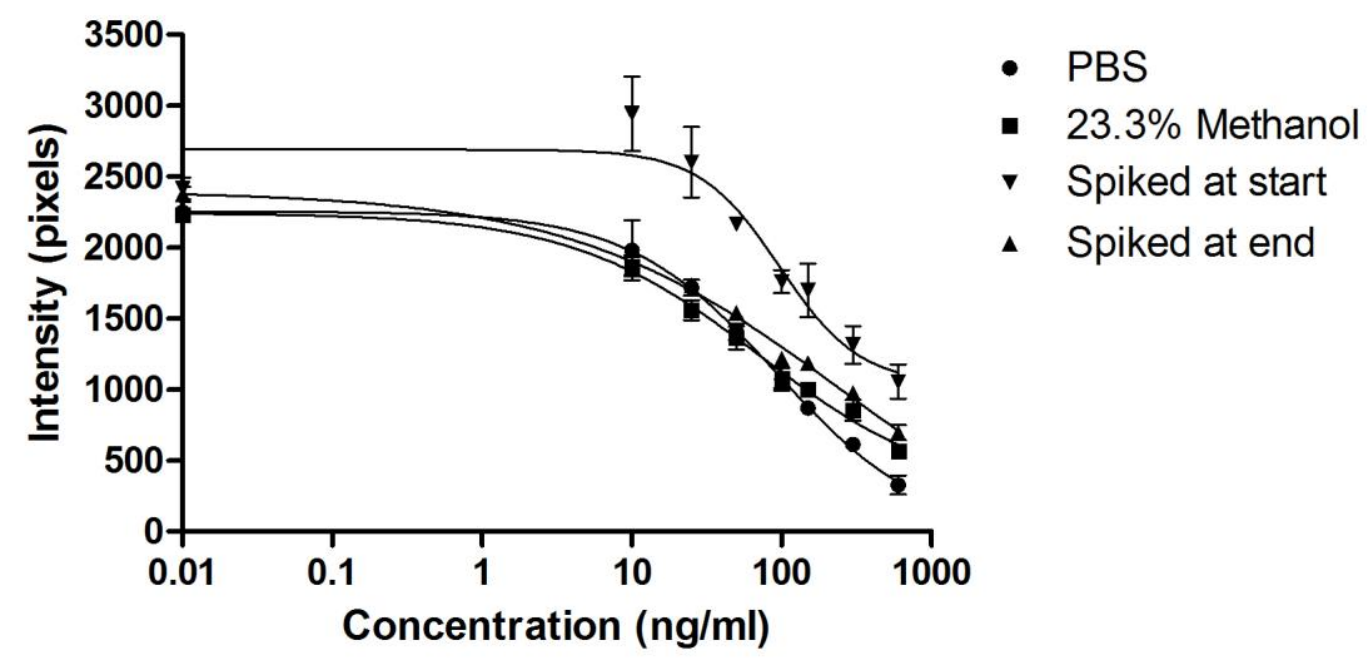

